\title{
AN INTRODUCTION TO EXCELLENT CLASSES
}

\author{
OLIVIER LESSMANN
}

\begin{abstract}
In this paper, we present Shelah's theory of excellence concluding with his categoricity theorem for the class of atomic models of a countable first order theory, under the assumption that there exists a large full model. This allows us to do the entire work within ZFC and without any assumption on the number of models of size $\aleph_{1}$, in contrast to Shelah's original treatment.
\end{abstract}

\section{INTRODUCTION}

The problem of categoricity has had a major influence on the development of model theory. A class is categorical in some cardinal $\lambda$ if all the models of the class of size $\lambda$ are isomorphic; the problem of categoricity is whether categoricity in some cardinals implies categoricity in others. In the first order case, Morley's solution to Łos conjecture [Mo], and Shelah's generalisation to uncountable languages [Sh 70] constitute the beginning of classification theory culminating in Shelah's Main Gap [Sh a], while Baldwin-Lachlan's solution [BaLa] laid the ground for geometric stability theory.

Categoricity for classes which are not first order is a considerably more complicated problem. It is a very active area with many partial results (see [Ke], [Sh 3], [KoSh], [MaSh], [Sh 48], [Sh 87a], and [Sh 87b], [Sh 88], [Sh 394], [ShVi], [Sh 576], [Sh 600], [Sh 705] to name but a few). Shelah views it as the most important problem in model theory and lists it first in [Sh 666]. He conjectures that, if the class $\mathcal{K}$ of models of a sentence in $L_{\omega_{1}, \omega}$ is categorical in some large enough cardinal, then it must be categorical in all large enough cardinals.

Historically, excellence arose after this conjecture was verified under the assumption that the class $\mathcal{K}$ contains sufficiently homogeneous models [Ke], [Sh 3]. This marked the beginning of classification for homogeneous model theory, as this context is now known. We have good notions of stability [Sh 3], [Sh 54], [GrLe], [Hy], superstability [HySh], [HyLe1], $\omega$-stability and total transcendence [Le1], and even simplicity [BuLe]. On the other hand, we have a Baldwin-Lachlan style theorem [Le1], as well as the beginning of geometric model theory [HLS]. At the time of [Ke], Keisler asked whether the existence of sufficiently homogeneous models actually followed from categoricity. Shelah answered negatively using an

Date: September 30, 2003. 
example of Markus [Ma], and introduced the theory of excellence. To do this, he first reduced the categoricity problem for the class of models of a sentence in $L_{\omega_{1}, \omega}$ to the categoricity of the class of atomic models of a countable first order theory [Sh 54]. This reduction is an important step (see Baldwin's paper in this volume for more details on it); it shows that proving the categoricity conjecture for $L_{\omega_{1}, \omega}$ is equivalent to proving it for the apparently simpler context of the class of atomic models of a countable first order theory. Then, in [Sh 87a], [Sh 87b], Shelah showed:

Theorem -1.1. Assume $2^{\aleph_{n}}<2^{\aleph_{n+1}}$ for each $n<\omega$. Let $\mathcal{K}$ be the class of atomic models of a countable first order theory and assume further that $\mathcal{K}$ has fewer than $2^{\aleph_{1}}$ nonisomorphic models of size $\aleph_{1}$.

(1) If $\mathcal{K}$ is categorical in each $\aleph_{n}$, for $n<\omega$, then $\mathcal{K}$ is excellent.

(2) If $\mathcal{K}$ is excellent and categorical in some uncountable cardinal, then $\mathcal{K}$ is categorical in all uncountable cardinals.

Modulo some additional properties, (1) thus shows that categoricity implies excellence, while (2) is the parallel to Morley's theorem for excellent classes. Above, (1) has the flavour of a nonstructure result and (2) belongs to structure theory.

In this paper, we focus on the structure part. We present a proof of (2), under the assumption that there exists a large full model (see below for more details). The existence of full models follows from excellence, and, for uncountable models, we can give an equivalent definition which makes sense for any class of atomic models. The reason for using a full model is that it allows us to present the entire treatment within ZFC and to remove the assumption on the number of uncountable models; for example, we obtain all the properties of independence directly from $\omega$-stability.

Solving the categoricity problem for excellent classes marked the beginning of classification theory in this context; Grossberg and Hart developed orthogonality calculus, introduced regular types, and proved the Main Gap for excellent classes [GrHa] (see the related article by Grossberg and Lessmann in this volume). We can also prove a Baldwin-Lachlan theorem, emphasising the role of quasiminimal types, and introduce a U-rank for types over models and obtain a picture very similar to the first order case [Le3]. Finally, quasiminimal types can be used to generalise Hrushovski's result [Hr] to the context of excellent classes [HLS], starting geometric stability theory proper. Excellence is the precursor to Shelah's work on good frames for the categoricity problem for abstract elementary classes [Sh 705].

Excellence appears naturally in several mathematical contexts. For example, it is the main dividing line when studying almost free algebras [MeSh] and it is also the key property in Zilber's work on complex exponentiation [Zi1] and [Zi2]. 
Let us now say a few words about fullness. Understanding which types are realised in the models of a class is a difficult problem as soon as the compactness theorem fails. For example, fix a homogeneous model $M$ of a first order theory $T$. There is no criterion to understand which types are realised inside $M$, except for complete types. We have weak compactness: A complete (first order) type $p \in$ $S(A)$, with $A \subseteq M$ and $|A|<\|M\|$, is realised in $M$ if and only if $p\lceil B$ is realised in $M$ for each finite subset $B$ of $M$. In full models, we have a similar condition but for complete types over models (at least when the full model is uncountable). An uncountable model $M$ of a countable first order theory $T$ is full, if $M$ realises every complete (first order) type $p \in S(N)$, where $N \prec M$ and $\|N\|<\|M\|$, provided that $p\lceil B$ is realised in $M$ for each finite $B \subseteq N$. This gives us a way of dealing with complete types over models.

In this paper, we consider the class $\mathcal{K}$ of atomic models of a countable first order theory. We will assume that there is a sufficiently large model $\mathfrak{C} \in \mathcal{K}$ which is full; we do not assume that every (small) model of $\mathcal{K}$ is in $\mathfrak{C}$ (this will be proved in the paper). We use $\mathfrak{C}$ twice in the course of the paper. The first time is to show that the categoricity of $\mathcal{K}$ in some uncountable cardinal implies that the class is $\omega$-stable, which means here that $\mathfrak{C}$ realises only countably many types over countable elementary submodels. The $\omega$-stability implies that $\mathcal{K}$ admits a bounded rank, which is then used to define an independence relation. We use $\mathfrak{C}$ a second time to prove the symmetry of the independence relation. Provided we restrict our attention to types over models, we prove that the independence relation satisfies all the properties of nonforking: symmetry, extension, transitivity, stationarity. This allows us to define independent systems of models and excellence; i.e. $\mathcal{K}$ is excellent if there exists a primary model over any $n$-dimensional independent system of countable models. We then prove some of the basic results of excellence, namely the existence of primary models over other sets. Finally, we present Shelah's categoricity theorem. At the end of the paper, we discuss Shelah's original approach and compare it with this presentation.

This paper grew out of lecture notes for a class on excellence that I gave at Oxford University in 2002 during the Michaelmas term. It assumes only basic model theory, say, up to Morley's theorem. For expositional purposes, a particular case of the general result is proved on two occasions, when the main idea is obscured by the additional technicality. We also streamline the text with comparisons to the relevant theorems of homogeneous model theory to illustrate both the differences and the limitations of the theorems, but familiarity with homogeneous model theory is not essential.

\section{TYPES AND $\omega$-STABILITY}

Fix a complete first order theory $T$ in a countable language $L$. In this paper, we consider the class $\mathcal{K}$ of atomic models of $T$, i.e. $M \in \mathcal{K}$ if and only if 
$M \models T$ and for any finite sequence $c \in M$, there exists a formula $\phi(x) \in L$ such that $\phi(x) \vdash \operatorname{tp}(c / \emptyset, M)$.

As usual, we work in a large sufficiently saturated model $\bar{M}$ of $T$ - which is not in $\mathcal{K}$. Satisfaction is defined with respect to $\bar{M}$. All sets and models are assumed to be inside $\bar{M}$ - so $\mathcal{K}$ is the class of atomic elementary submodels of $\bar{M}$. We use uppercase letters $A, B, C$ for sets, $M, N$ for models, and lowercase letters $a, b, c$ for finite sequences. We write $A B$ for the union of $A$ and $B$ and $A c$ for the union of $A$ with the range of the sequence $c$.

We first make a few observations about the class $\mathcal{K}$. The next remark shows that $(\mathcal{K}, \prec)$, where $M \prec N$ if $M$ is an elementary submodel of $N$, is an abstract elementary class (see Grossberg and Lessmann's paper in this volume for a definition). The proofs are left to the reader.

Remark 0.1. Let $\mathcal{K}$ be the class of atomic models of the countable theory $T$.

(1) $\left(L S(\mathcal{K})=\aleph_{0}\right)$ If $A \subseteq M \in \mathcal{K}$, then there exists $N \prec M, A \subseteq N$, such that $\|N\|=|A|+\aleph_{0}$. Since $N \prec M$ and $M \in \mathcal{K}$, then $N \in \mathcal{K}$.

(2) (Tarski-Vaught's chain condition) If $\left(M_{i}: i<\alpha\right)$ is an increasing and continuous elementary chain of models such that $M_{i} \in \mathcal{K}$ for each $i<\alpha$, then $\bigcup_{i<\alpha} M_{i} \in \mathcal{K}$. Furthermore $M_{0} \prec \bigcup_{i<\alpha} M_{i}$ and if $M_{i} \prec N \in \mathcal{K}$ for each $i<\alpha$ then also $\bigcup_{i<\alpha} M_{i} \prec N$.

Recall that $M$ is $\lambda$-homogeneous if for any elementary $f: M \rightarrow M$ of size less than $\lambda$ and $a \in M$ there exists $g: M \rightarrow M$ extending $f$ such that $a \in \operatorname{dom}(g)$. We say that $M$ is homogeneous if $M$ is $\|M\|$-homogeneous.

Remark 0.2. (1) Each model of $\mathcal{K}$ is $\omega$-homogeneous, and therefore embeds elementarily any countable atomic set.

(2) There is a unique countable model in $\mathcal{K}$.

We now consider the problem of types. As usual, we denote by $S(A)$ the set of complete $L$-types over $A$ in finitely many variables. In the first order case, all types are realisable by models of a theory; this is an important consequence of the compactness theorem. In our context, the situation is a little more delicate; if $A \subseteq M \in \mathcal{K}$ and if $p \in S(A)$ is realised in $M$ by, say, $c$, then $A \cup c$ is an atomic set. This gives us a necessary condition for which types are realisable in the models of our class. We make the following definition:

Definition 0.3. We let $\mathrm{S}_{\mathrm{at}}(A)$ be the set of types $p \in S(A)$ such that for all $c \models p$, the set $A c$ is atomic.

When $A$ is not atomic, $\mathrm{S}_{\text {at }}(A)$ is clearly empty. Also, if $A c$ is atomic for some $c \models p$, then $A d$ is atomic for any $d \models p$. Furthermore, if $\left(A_{i}: i<\alpha\right)$ is an increasing and continuous sequence of atomic sets, and $\left(p_{i}: i<\alpha\right)$ is an 
increasing and continuous sequence of types, with $p_{i} \in \mathrm{S}_{\mathrm{at}}\left(A_{i}\right)$ for $i<\alpha$, then the type $p=\bigcup_{i<\alpha} p_{i} \in \mathrm{S}_{\mathrm{at}}\left(\bigcup_{i<\alpha} A_{i}\right)$.

However, given a (partial) type $p$ over an atomic set $A$ (indeed, even for a complete type $p \in \mathrm{S}_{\mathrm{at}}(B)$, where $B$ is a subset of $A$ ), there may not exist $q \in$ $\mathrm{S}_{\text {at }}(A)$ extending $p$. This may fail also for countable $A$.

Another problem is that, in general, there may be $A \subseteq M \in \mathcal{K}$ and $p \in$ $\mathrm{S}_{\text {at }}(A)$ not realised in any $N \in \mathcal{K}$. This only occurs for uncountable $A$, though: If $c \in \bar{M}$ realises $p \in \mathrm{S}_{\text {at }}(A)$, then $A c$ is atomic by definition. If $A$ is countable, then $A c$ embeds elementarily inside the countable model $M_{0}$ of $\mathcal{K}$. This embedding extends in $\bar{M}$ to an elementary map whose range contains $M_{0}$. The image of $M_{0}$ under the inverse of this map produces an atomic model containing $A c$. It follows that, for countable $A$, the set $\mathrm{S}_{\text {at }}(A)$ corresponds exactly to the set of types over $A$ realised by models in $\mathcal{K}$.

The fact that, for $A$ uncountable, some types in $\mathrm{S}_{\text {at }}(A)$ will be omitted is unavoidable unless there exists an $\left(|A|+\aleph_{0}\right)$-homogeneous model in $\mathcal{K}$ of size at least $|A|+\aleph_{0}$. But, as Shelah showed, there are uncountably categorical atomic classes $\mathcal{K}$ not containing any uncountable $\omega_{1}$-homogeneous model. So, outside of homogeneous model theory, types over general sets are intractable. In this paper, we will deal essentially with types over models. We make the following hypothesis throughout the text.

Hypothesis 0.4. There exists a model $\mathfrak{C} \in \mathcal{K}$ of size at least $\bar{\kappa}$, for some suitably large cardinal $\bar{\kappa}$, with the property that if $p \in \mathrm{S}_{\text {at }}(M)$ and $M \prec \mathfrak{C}$ of size less than $\bar{\kappa}$, then $p$ is realised in $\mathfrak{C}$.

In this paper, 'suitably large' means that $\bar{\kappa}$ is assumed to be at least the categoricity cardinal, and at least the Hanf number for atomic classes $\left(=\beth_{\omega_{1}}\right)$. This latter condition ensures, in particular, that $\mathcal{K}$ has arbitrarily large models. We will see in subsequent sections that $\mathfrak{C}$ is full. The existence of full models follows from excellence; by introducing them early, we can present the entire theory within ZFC in a very smooth way. The existence of full models does not imply the existence of homogeneous models. Notice also that we do not assume that all (small) models of $\mathcal{K}$ embed in $\mathfrak{C}$ (this will follow from excellence); for now, it is enough that all countable models of $\mathcal{K}$ embed into $\mathfrak{C}$, which is a consequence of $\omega$-homogeneity.

We now consider $\omega$-stability. There are several possible notions for $\omega$ stability which we discuss below; some are equivalent, some are not.

Definition 0.5. The class $\mathcal{K}$ is $\lambda$-stable if $\left|\mathrm{S}_{\text {at }}(M)\right| \leq \lambda$, for each $M \in \mathcal{K}$ of size $\lambda$.

Proposition 0.6. The following conditions are equivalent:

(1) $\mathcal{K}$ is $\omega$-stable.

(2) $\mathfrak{C}$ realises only countably many types over countable subsets. 
(3) Each $M \in \mathcal{K}$ realises only countably many types over countable subsets.

Proof. (3) implies (2) is clear. To see that (2) implies (1), suppose that $\mathrm{S}_{\mathrm{at}}(M)$ is uncountable for some countable $M \in \mathcal{K}$. By $\omega$-homogeneity, we may assume that $M \prec \mathfrak{C}$, and thus $\mathfrak{C}$ is not $\omega$-stable since it realises each type in $\mathrm{S}_{\text {at }}(M)$. For (1) implies (3), if $M \in \mathcal{K}$ realises uncountably many types over a countable subset, then it realises uncountably many types over a countable submodel $M_{0} \prec N$. Thus $\mathrm{S}_{\mathrm{at}}\left(M_{0}\right)$ is uncountable, contradicting (1).

$\mathcal{K}$ can be $\omega$-stable while $T$ is unstable: Consider the countable theory in the language $\{N,+, 0,1\}$, where $T$ has PA on the predicate $N$ and asserts that the complement of $N$ is infinite. $T$ is unstable since it has the strict order property. However, the class $\mathcal{K}$ of atomic models of $T$ has arbitrarily large homogeneous models (hence satisfies our hypothesis 0.4 ), and is $\omega$-stable.

Without additional assumptions, the $\omega$-stability of $\mathcal{K}$ does not even imply that $\mathrm{S}_{\text {at }}(A)$ is countable for each countable atomic $A$ : although each type $p \in$ $\mathrm{S}_{\mathrm{at}}(A)$ is realisable inside a model, there may be no model realising jointly all types in $\mathrm{S}_{\mathrm{at}}(A)$. If we had an uncountable $\omega_{1}$-homogeneous model, we could do this (or amalgamation over sets, which is the same); it turns out to be equivalent, as is shown in the following fact [Le2]. The existence of countable sets $A$ with $\mathrm{S}_{\text {at }}(A)$ uncountable is a core difference with the categoricity problem in the homogeneous case. It is the basic motivation behind excellence and will be revisited in Section 2.

Fact 0.7 (Lessmann). Suppose that $\mathrm{S}_{\mathrm{at}}(A)$ is countable for each countable atomic set $A$. If $\mathcal{K}$ has an uncountable model, then $\mathcal{K}$ has arbitrarily large homogeneous models,

Throughout this paper, we will make occasional use of the following fact, often referred to as Morley's methods.

Fact 0.8. Suppose $\left(a_{i}: i<\beth_{\omega_{1}}\right) \subseteq M \in \mathcal{K}$. Let $L^{*}$ be an expansion of $L$ with Skolem functions and $T^{*}$ be the theory of $\mathfrak{C}$ is this expansion. Then there exists an $L^{*}$-indiscernible sequence $\left(b_{i}: i<\omega\right)$ such that for each $n<\omega$ we can find $i_{0}<\cdots<i_{n}<\beth_{\omega_{1}}$ satisfying

$$
\operatorname{tp}_{L^{*}}\left(b_{0}, \ldots, b_{n} / \emptyset\right)=\operatorname{tp}_{L^{*}}\left(a_{i_{0}}, \ldots, a_{i_{n}} / \emptyset\right) .
$$

It follows that the reduct to $L$ of the Skolem Hull of $\left(b_{i}: i<\omega\right)$ is a model of $\mathcal{K}$.

Recall that $\mathcal{K}$ is $\lambda$-categorical for a cardinal $\lambda$ if all models of $\mathcal{K}$ of size $\lambda$ are isomorphic. $\mathcal{K}$ is always $\aleph_{0}$-categorical. We now connect categoricity with $\omega$-stability.

Proposition 0.9. If $\mathcal{K}$ is $\lambda$-categorical for some uncountable $\lambda$, then $\mathcal{K}$ is $\omega$-stable. 
Proof. Suppose that $\mathcal{K}$ is not $\omega$-stable. Then $\mathfrak{C}$ realises uncountably many types over a countable subset. By the Downward-Löwenheim theorem, we can find $M \prec$ $\mathfrak{C}$ of size $\lambda$ realising uncountably many types over a countable subset. On the other hand, since $\mathcal{K}$ has arbitrarily large models, we can use Fact 0.8 to find an infinite $L^{*}$-indiscernible sequence $\left(b_{i}: i<\omega\right)$ inside some $N \in \mathcal{K}$. By compactness, we can extend $\left(b_{i}: i<\omega\right)$ to a dense linearly ordered $L^{*}$-indiscernible sequence $I$ of size $\lambda$. By construction, the Skolem Hull of $I$ also omits all the nonisolated types of $T$ (a counterexample would otherwise provide one in $N$ ), and hence its reduct is a model in $\mathcal{K}$. It is easy to see that this reduct is $\omega$-stable of size $\lambda$, which contradicts $\lambda$-categoricity.

Shelah's example to answer Keisler's question negatively shows that uncountable categoricity does not necessarily imply that $\mathrm{S}_{\mathrm{at}}(A)$ is countable for all countable atomic $A$.

\section{RANK AND INDEPENDENCE}

From now on, until the rest of the paper, we assume that $\mathcal{K}$ is $\omega$-stable. In this section, we introduce a rank. The rank is bounded in the $\omega$-stable case, and equality of ranks provides an independence relation which we show to be wellbehaved over models of $\mathcal{K}$.

Definition 1.1. For any formula $\phi(x)$ with parameters in $M \in \mathcal{K}$, we define the $\operatorname{rank} R_{M}[\phi]$. The rank $R_{M}[\phi]$ will be an ordinal, -1 , or $\infty$ and we have the usual ordering $-1<\alpha<\infty$ for any ordinal $\alpha$. We define the relation $R_{M}[\phi] \geq \alpha$ by induction on $\alpha$.

(1) $R_{M}[\phi] \geq 0$ if $\phi$ is realised in $M$;

(2) $R_{M}[\phi] \geq \delta$, when $\delta$ is a limit ordinal, if $R_{M}[\phi] \geq \alpha$ for every $\alpha<\delta$;

(3) $R_{M}[\phi] \geq \alpha+1$ if the following two conditions hold:

(a) There is $a \in M$ and a formula $\psi(x, y)$ such that

$$
R_{M}[\phi(x) \wedge \psi(x, a)] \geq \alpha \quad \text { and } \quad R_{M}[\phi(x) \wedge \neg \psi(x, a)] \geq \alpha ;
$$

(b) For every $c \in M$ there is a formula $\chi(x, c)$ isolating a complete type over $c$ such that

$$
R_{M}[\phi(x) \wedge \chi(x, c)] \geq \alpha .
$$

We write:

$R_{M}[\phi]=-1$ if $\phi$ is not realised in $M$;

$R_{M}[\phi]=\alpha$ if $R_{M}[\phi] \geq \alpha$ but it is not the case that $R_{M}[\phi] \geq \alpha+1$;

$R_{M}[\phi]=\infty$ if $R_{M}[\phi] \geq \alpha$ for every ordinal $\alpha$.

For any set of formulas $p(x)$ over $A \subseteq M$, we let

$$
R_{M}[p]=\min \left\{R_{M}[\phi] \mid \phi=\wedge q, q \subseteq p, p \text { finite }\right\} .
$$



model of $\mathcal{K}$.

Note that the condition $R[p] \geq 0$ does not imply that $p$ is realised in a

We first write down a few properties of the rank. They are all basic and can be proved easily by induction or directly. (7) follows from (6) and (1) using the countability of the language $L$.

Lemma 1.2. (1) $R_{M}[\phi(x, b)]$ depends on $\phi(x, y)$ and $\operatorname{tp}(b / \emptyset)$ only.

(2) If $p$ is over $M$ and $N$, for $M, N \in \mathcal{K}$, then $R_{M}[p]=R_{N}[p]$.

(3) If $p$ is finite and $\phi$ is obtained by taking the conjunction of all the formulas in $p$, then $R[p]=R[\phi]$.

(4) (Finite Character) For each $p$ there is a finite subset $q$ of $p$ such that $R[p]=$ $R[q]$.

(5) (Monotonicity) If $p \subseteq q$, then $R[p] \geq R[q]$.

(6) If $R[p]=\alpha$ and $\beta<\alpha$ there is $q$ such that $R[q]=\beta$.

(7) There exists an ordinal $\alpha_{0}<\omega_{1}$, such that if $R[p] \geq \alpha_{0}$ then $R[p]=\infty$.

In view of (2), we will drop the subscript $M$. We now show that $\omega$-stability implies that the rank is bounded (the converse is Proposition 1.6). The idea of the proof is essentially like the first order case: we construct of binary tree of formulas, whose branches give us continuum many types. There is one difference: to contradict $\omega$-stability, we need the types to be in $\mathrm{S}_{\text {at }}(M)$ for some countable $M \in \mathcal{K}$. To achieve this, we simply choose isolating formulas along the way, and force the parameters to enumerate a model.

Theorem 1.3. $R[p]<\infty$ for every type $p$.

Proof. We prove the contrapositive. Suppose there is a type $p$ over some atomic model $M$ such that $R[p]=\infty$. We may assume that $p=\{\phi(x, b)\}$ is a formula and also that $M$ is countable by the previous lemma. Let $\left\{a_{i}: i<\omega\right\}$ be an enumeration of $M$.

We construct formulas $\phi_{\eta}\left(x, b_{\eta}\right)$, for $\eta \in{ }^{<\omega} 2$, such that:

(1) $\phi_{\eta}\left(x, b_{\eta}\right)$ isolates a complete type over $b_{\eta}$;

(2) $\models \forall x\left[\phi_{\nu}\left(x, b_{\nu}\right) \rightarrow \phi_{\eta}\left(x, b_{\eta}\right)\right]$ when $\eta<\nu$;

(3) $\phi_{\eta^{\wedge} 0}$ and $\phi_{\eta^{\wedge} 1}$ are contradictory;

(4) $R\left[\phi_{\eta}\right]=\infty$;

(5) $a_{\ell(\eta)} \in b_{\eta}$

This is possible: The construction is by induction on $\ell(\eta)$.

For \langle\rangle : Since $R[\phi]=\infty$, in particular $R[\phi] \geq \omega_{1}+1$ so there exists $\phi_{\langle\rangle}$ over $b a_{0}$ isolating a complete type over $b a_{0}$ such that $R\left[\phi \wedge \phi_{\eta}\right] \geq \omega_{1}$. The formula $\phi_{\langle\rangle}$is as required. 
Assume that we have constructed $\phi_{\eta}\left(x, b_{\eta}\right)$ with $\ell(\eta)<\omega$. Since $R\left[\phi_{\eta}\right]=$ $\infty$, in particular $R\left[\phi_{\eta}\right] \geq\left(\omega_{1}+1\right)+1$. Hence, there is $c \in M$ and $\psi(x, y)$ such that

$$
R\left[\phi_{\eta} \wedge \psi(x, c)\right] \geq \omega_{1}+1 \quad \text { and } \quad R\left[\phi_{\eta} \wedge \neg \psi(x, c)\right] \geq \omega_{1}+1 .
$$

Let $b_{\eta \wedge 0}=b_{\eta \wedge 1}=c b_{\eta} a_{\ell(\eta)+1}$. By (*) and the definition of the rank ((3)(b)), there are $\phi_{\eta^{\wedge} \ell}\left(x, b_{\eta^{\wedge} \ell}\right)$ isolating a complete type over $b_{\eta^{\wedge} \ell}$ for $\ell=0,1$, such that

$$
R\left[\phi_{\eta} \wedge \psi(x, c) \wedge \phi_{\eta^{\wedge} 0}\left(x, b_{\eta^{\wedge} 0}\right)\right] \geq \omega_{1}
$$

and

$$
R\left[\phi_{\eta} \wedge \neg \psi(x, c) \wedge \phi_{\eta^{\wedge} 1}\left(x, b_{\eta^{\wedge} 1}\right)\right] \geq \omega_{1}
$$

Then $\phi_{\eta^{\wedge} \ell}\left(x, b_{\eta^{\wedge} \ell}\right)$ are as required, for $\ell=0,1$.

This is enough: For each $\eta \in{ }^{\omega} 2$, define $p_{\eta}:=\bigcup_{n \in \omega} p_{\eta\lceil n}$. Notice that each $p_{\eta}$ determines a complete type over $M$ with the property that $M c_{\eta}$ is atomic for any realisation $c_{\eta}$ of $p_{\eta}$. Hence, each $p_{\eta} \in \mathrm{S}_{\text {at }}(M)$, so $\mathrm{S}_{\text {at }}(M)$ has size continuum, which contradicts the $\omega$-stability of $\mathcal{K}$.

Recall that $p \in \mathrm{S}_{\text {at }}(A)$ splits over $B \subseteq A$ if there exist $c, d \in A$ realising the same type over $B$ and $\phi(x, y)$ such that $\phi(x, c) \in p$ and $\neg \phi(x, d) \in p$. The next proposition examines the connection between the rank and nonsplitting. It also shows that we may have at most one same rank extension over a model.

Proposition 1.4. If $p \in \mathrm{S}_{\mathrm{at}}(M), M \in \mathcal{K}$ and $\phi(x, b) \in p$ such that $R[p]=$ $R[\phi(x, b)]$. Then $p$ does not split over $b$. Furthermore, $p$ is the only type in $\mathrm{S}_{\mathrm{at}}(M)$ extending $\phi(x, b)$ with the same rank.

Proof. Suppose that $p$ splits over $b$. Let $\psi(x, y)$, and $c, d \in M$ such that $\operatorname{tp}(c / b)=$ $\operatorname{tp}(d / b)$ and $\psi(x, d) \in p$ and $\neg \psi(x, d) \in p$. Then $R[\phi(x, b) \wedge \psi(x, c)] \geq \alpha$, and $R[\phi(x, b) \wedge \neg \psi(x, d)] \geq \alpha$ by monotonicity. By Lemma 1.2, we also have $R[\phi(x, b) \wedge \psi(x, d)] \geq \alpha$, so (a) of the rank is satisfied at the successor stage. Now let $e \in M$. By monotonicity, $R[p\lceil b c] \geq \alpha$. There exists $\chi \in p\lceil b c$ isolating $p\lceil b c$. By monotonicity, $R[\phi \wedge \chi] \geq \alpha$, so (b) of the rank is satisfied at the successor stage. Hence $R[\phi] \geq \alpha+1$, a contradiction.

For uniqueness, suppose that $q \neq p \in S_{a t}(M)$ extend $\phi(x, b)$. Suppose $\psi(x, c) \in q$ such that $\neg \psi(x, c) \in p$. By monotonicity, we have $R[\phi(x, b) \wedge$ $\psi(x, c)] \geq \alpha$ and $R[\phi(x, b) \wedge \neg \psi(x, c)] \geq \alpha$, so (a) of the rank is satisfied. It is easy to see that (b) of the rank is satisfied the same way as the previous paragraph

The proposition shows that if $p \in \mathrm{S}_{\text {at }}(M)$ then $p$ does not split over a finite set. This is not true for $p \in \mathrm{S}_{\text {at }}(A)$ in general.

Proposition 1.5. Let $p \in \mathrm{S}_{\mathrm{at}}(M), M \in \mathcal{K}$ and let $\phi(x, b) \in p$ be such that $R[p]=R[\phi]$. Let $C$ be an atomic set containing $M$, then there exists a unique $q \in \mathrm{S}_{\mathrm{at}}(C)$ extending $\phi$ (and therefore $p$ ) such that $R[p]=R[q]$. 
Proof. Let $\phi(x, b) \in p$ such that $R[p]=R[\phi]$. By the previous proposition, $p$ does not split over $b$. Let $q$ be the following set of formulas

$$
\left\{\psi(x, c): c \in C, \psi(x, y) \in L \text {, there exists } c^{\prime} \in M \text { realising } \operatorname{tp}(c / b)\right\} .
$$

Since $p$ does not split over $b$, this is well-defined. Similarly, this determines a type $q \in \mathrm{S}_{\mathrm{at}}(C)$. It is easy to check that $q$ does not split over $b$ and has the same rank as $p$.

For uniqueness, suppose that $R\left[q_{\ell}\right]=R[\phi]$, for $\ell=1,2$ both contain $\phi$. Then $R\left[q_{\ell}\lceil M]=R[p]\right.$ for $\ell=1,2$ be the previous proposition. Then $q_{1}=q_{2}$ since both do not split over $b$.

We now prove the converse to Theorem 1.3. Together, they form a particular case of the stability spectrum theorem; $\omega$-stability implies $\lambda$-stability for all $\lambda$.

Proposition 1.6. If $R[p]<\infty$ for all types $p$, then $\mathcal{K}$ is $\lambda$-stable, for each infinite $\lambda$.

Proof. Let $M \in \mathcal{K}$. Let $p \in \mathrm{S}_{\text {at }}(M)$ and let $\phi(x, b) \in p$ such that $R[p]=$ $R[\phi(x, b)]$. Since $p$ is the only extension of $\phi(x, b)$ with the same rank, the number of types in $\mathrm{S}_{\mathrm{at}}(M)$ is at most the number of formulas over $M$, which is $\|M\|$.

In general, a type may fail to have an extension to a larger set, so in particular, it may fail to have a same rank extension. This is why we consider stationary types.

Definition 1.7. We say that $p \in \mathrm{S}_{\text {at }}(A)$ is stationary if there exists $b \in A$, $\phi(x, b) \in p$, and $M \in \mathcal{K}$ containing $A$ with $q \in \mathrm{S}_{\mathrm{at}}(M), \phi(x, b) \in q$ and $R[\phi(x, b)]=R[p]=R[q]$.

Clearly, complete types over models are stationary. The previous proposition does not assert that the nonsplitting extension is actually realised in a model (it is, if we work inside $\mathfrak{C}$ ). It only says that when $p \in \mathrm{S}_{\text {at }}(A)$ is stationary and $C$ is atomic containing $A$, there is a unique extension in $\mathrm{S}_{\text {at }}(C)$ of the same rank. We will denote this extension by $p \uparrow C$.

We now introduce fullness, a substitute for saturation. Full is called weakly full in [Sh 87a].

Definition 1.8. A model $M \in \mathcal{K}$ is $\lambda$-full over $A \subseteq M$ if for all stationary $p \in$ $\mathrm{S}_{\text {at }}(B)$ with $B \subseteq M$ finite, and for all $C \subseteq M$ of size less than $\lambda, M$ realises $p\lceil A B C$. We say that $M$ is $\lambda$-full if $M$ is $\lambda$-full over each subset of size less than $\lambda$. Finally, we say that $M$ is full if $M$ is $\lambda$-full for $\lambda=\|M\|$.

Note that if $M$ is $\lambda$-full then $M$ realises each $p \in \mathrm{S}_{\text {at }}(N)$ for $N \prec M$ of size less than $\lambda$. Also, if $M$ is $\lambda$-full over $A$, then it is $\lambda$-full over $B$, for any 
$B \subseteq A$. We have the following easy proposition, which is left to the reader. It shows that $\mathfrak{C}$ is $\bar{\kappa}$-full:

Proposition 1.9. Let $M \in \mathcal{K}$ be uncountable. The following are equivalent;

(1) $M$ is $\lambda$-full.

(2) $M$ realises each $p \in \mathrm{S}_{\text {at }}(N)$, with $N \prec M$ of size less than $\lambda$.

We are going to prove Symmetry over models. For this, we will use the order property which was introduced by Shelah in [Sh 12].

Definition 1.10. We say that $\mathcal{K}$ has the order property if there exist a model $M \in$ $\mathcal{K}$, a formula $\phi(x, y) \in L$ and $\left(d_{i}: i<\beth_{\omega_{1}}\right) \subseteq M$ such that

$$
M \models \phi\left(d_{i}, d_{j}\right) \quad \text { if and only if } \quad i<j .
$$

In the definition above, it is equivalent to ask for arbitrarily long orders (use Morley's methods). It is a familiar theorem in the first order case that the order property contradicts stability. It holds at this level of generality also - but it is necessary to have arbitrarily long orders. We present the following particular case suitable for our purposes.

Proposition 1.11. $\mathcal{K}$ does not have the order property.

Proof. Suppose that $\mathcal{K}$ has the order property. Let $\left(d_{i}: i<\beth_{\omega_{1}}\right) \subseteq M \in \mathcal{K}$ such that $M \models \phi\left(d_{i}, d_{j}\right)$ if and only if $i<j$. By Fact 0.8 , there exists $\left(b_{n}: n<\omega\right)$ $L^{*}$-indiscernible such that $=\phi\left(b_{n}, b_{m}\right)$ if and only if $n<m$, and furthermore, the reduct to $L$ of the Skolem Hull of $\left(b_{n}: n<\omega\right)$ is in $\mathcal{K}$. By compactness, we can find $\left(b_{i}: i \in \mathbb{R}\right) L^{*}$-indiscernible such that any finite subsequence of it satisfies the same $L^{*}$-type as any finite subsequence of $\left(b_{n}: n<\omega\right)$ of the same length. Let $N$ be the reduct to $L$ of the Skolem Hull of $\left(b_{i}: i \in \mathbb{R}\right)$. By construction $N \in \mathcal{K}$ (it is a model of $T$ and must be atomic since a counterexample can be used to provide a counterexample in $M)$. Furthermore $N \models \phi\left(b_{i}, b_{j}\right)$ if and only if $i<j$. Letting $B=\bigcup_{i \in \mathbb{Q}} b_{i}$ gives us a countable subset of $N$ over which $2^{\aleph_{0}}$ types are realised by density of $\mathbb{Q}$. This contradicts the $\omega$-stability of $\mathcal{K}$ (Proposition 0.6).

We can now prove the symmetry property of the rank.

Proposition 1.12. (Symmetry) Let $a, c$ and $M \in \mathcal{K}$ be such that $M a c$ is atomic. Then $R[\operatorname{tp}(a / M c)]=R[\operatorname{tp}(a / M)]$ if and only if $R[\operatorname{tp}(c / M a)]=R[\operatorname{tp}(c / M)]$.

Proof. Notice that by Finite Character and Monotonicity, we may assume that $M$ is countable, and so by $\omega$-homogeneity of $\mathfrak{C}$, we may assume that $a, c$ and $M$ are inside $\mathfrak{C}$. Suppose the conclusion of the proposition fails; we will contradict the $\omega$-stability of $\mathcal{K}$ by showing that it has the order property. We can choose a formula $\psi(x, y)$ over $M$ such that

$$
R[\operatorname{tp}(a / M c)]=R[\psi(x, c)]=R[\operatorname{tp}(a / M)]
$$


while

$$
R[\operatorname{tp}(c / M a)]=R[\psi(a, y)]<R[\operatorname{tp}(c / M)] .
$$

Define $\left(a_{i}, c_{i}: i<\beth_{\omega_{1}}\right) \subseteq \mathfrak{C}$ and $B_{i}=M \cup\left\{a_{j}, c_{j}: j<i\right\}$ such that

(1) $c_{i} \in \mathfrak{C}$ realises $\operatorname{tp}(c / M)$ and $R\left[\operatorname{tp}\left(c_{i} / B_{i}\right)\right]=R[\operatorname{tp}(c / M)]$,

(2) $a_{i} \in N$ realises $\operatorname{tp}(a / M)$ and $R\left[\operatorname{tp}\left(a_{i} / B_{i} c_{i}\right)=R[\operatorname{tp}(a / M)]\right.$.

This is possible since both $\operatorname{tp}(a / M)$ and $\operatorname{tp}(c / M)$ are stationary and thus the realisations $a_{i}$ and $c_{i}$ in (1) and (2) of the unique nonsplitting extensions of these types exist by fullness of $\mathfrak{C}$.

This implies the order property: Suppose that $i>j$. Then $a_{j} \in B_{i}$. If $=\psi\left(a_{j}, c_{i}\right)$, then $R\left[\operatorname{tp}\left(c_{i} / B_{i}\right)\right] \leq R\left[\psi\left(a_{i}, y\right)\right]=R[\psi(a, y)]<R\left[\operatorname{tp}\left(c_{i} / B_{i}\right)\right]$ contradicting (1) (we used the fact that $\operatorname{tp}\left(a_{i} / M\right)=\operatorname{tp}(a / M)$ to see that the middle equality holds). Hence $\models \neg \psi\left(a_{j}, c_{i}\right)$ if $i>j$.

Now if $i \leq j$, then we have $\operatorname{tp}\left(a_{j} / M c\right)=\operatorname{tp}(a / M c)$, by uniqueness of same rank extensions, so $\models \psi\left(a_{j}, c\right)$. Since $\operatorname{tp}\left(a_{j} / B_{j} c_{j}\right)$ does not split over $B$ by (2) and $\operatorname{tp}(c / M)=\operatorname{tp}\left(c_{i} / M\right)$, we must have $=\psi\left(a_{j}, c_{i}\right)$.

Thus, $\phi\left(x_{1}, x_{2} ; y_{1}, y_{2}\right):=\psi\left(x_{1}, y_{2}\right)$ and $d_{i}:=c_{i} a_{i}$, for $i<\beth_{\omega_{1}}$, witness the order property.

We can now define a natural independence relation using the rank: For $A, B, C$ such that $A \cup B \cup C$ is atomic, we write

$$
A \underset{B}{\downarrow} C \quad \text { if } \quad R[\operatorname{tp}(a / B C)]=R[\operatorname{tp}(a / B)], \text { for all } a \in A .
$$

We will say that $A$ is free from $C$ over $B$ if $A \underset{B}{\downarrow} C$. We now gather the properties $B$

we have established for this dependence relation. The reader used to the first order case may wonder whether (6) and (8) hold with sets instead of models. The answer is no in general.

Proposition 1.13. Assume $A, B, C, D$ are sets whose union is atomic.

(1) (Invariance) If $f$ is an elementary map, then

$$
A \underset{B}{\downarrow} C \text { if and only if } f(A) \underset{f(B)}{\downarrow} f(C) .
$$

(2) (Monotonicity) If $A^{\prime} \subseteq A$ and $C^{\prime} \subseteq C \cup B$ and $A \underset{B}{\downarrow} C$, then $A^{\prime} \underset{B}{\downarrow} C^{\prime}$.

(3) (Finite Character)

$$
A \underset{B}{\underset{B}{\downarrow} C} \text { if and only if } \quad A^{\prime} \underset{B}{\downarrow} C^{\prime},
$$

for each finite $A^{\prime} \subseteq A$ and finite $C^{\prime} \subseteq C$. 
(4) (Transitivity) If $B \subseteq C \subseteq D$, then

$$
A \underset{B}{\downarrow} C \text { and } A \underset{C}{\downarrow} D \text { if and only if } A \underset{B}{\downarrow} D .
$$

(5) (Local Character) If $a \cup C$ is atomic, then there exists a finite $B \subseteq C$ such that $a \underset{B}{\downarrow} C$.

(6) (Extension over models) Let $A \cup M$ be atomic, $M \in \mathcal{K}$ and $C$ be atomic containing $A$. Then there exists $a^{\prime}$ realising $\operatorname{tp}(a / M)$ such that $a^{\prime} \cup C$ is atomic and $a^{\prime} \downarrow C$.

$$
M
$$

(7) (Stationarity over models) Suppose that $a_{\ell}$ realises $\operatorname{tp}(a / M), a_{\ell} \cup C$ is atomic, and $a_{\ell} \underset{M}{\downarrow} C$ for $\ell=1,2$. Then $\operatorname{tp}\left(a_{1} / C\right)=\operatorname{tp}\left(a_{2} / M\right)$.

(8) (Symmetry over models) If $A \cup C \cup M$ is atomic, then

$$
A \underset{M}{\downarrow} C \text { if and only if } C \underset{M}{\downarrow} A \text {. }
$$

In the sequel, we will use these properties extensively; on occasions, we will simply say 'by independence calculus' when establishing the independence of certain sets from others by using a sequence of these properties.

\section{GOOD SETS, PRIMARY, AND FULL MODELS}

Recall that $\mathcal{K}$ is $\omega$-stable. In order to define excellence, we will also need primary models.

Definition 2.1. We say that $M \in \mathcal{K}$ is primary over $A$, if $M=A \cup\left\{a_{i}: i<\alpha\right\}$, and for each $i<\alpha$ the type $\operatorname{tp}\left(a_{i} / A \cup\left\{a_{j}: j<i\right\}\right)$ is isolated.

The sequence $\left(a_{i}: i<\alpha\right)$ is referred to as a construction of $M$ over $A$. It is a standard fact that if $M \in \mathcal{K}$ is primary over $A$ then for each $c \in M$, the type $\operatorname{tp}(c / A)$ is isolated. If $M$ is primary over $A$, then it is easy to see that it is prime over $A$. Recall that a model $M \in \mathcal{K}$ is prime over $A$, if for each $N \in \mathcal{K}$ containing $A$, there is an elementary map $f: M \rightarrow N$ which is the identity on $A$.

The main tool for producing primary models over countable sets is the following corollary to Henkin's omitting type theorem:

Fact 2.2. Let $T$ be a countable theory. Assume that for each consistent formula $\phi(x)$ there exists a complete type over the empty set containing $\phi(x)$ which is isolated. Then there exists a countable atomic model of $T$.

This leads to the next definition, important mostly for countable sets.

Definition 2.3. An atomic set $A$ is $\operatorname{good}$ if for each $\phi(x, a)$ with $a \in A$ and $=\exists x \phi(x, a)$, there is a complete type $p \in \mathrm{S}_{\text {at }}(A)$ containing $\phi(x, a)$ which is isolated. 
The next lemma is the motivation behind the definition of good sets.

Lemma 2.4. Let $A$ be countable and atomic. If $A$ is good, then there is a primary model over $A$.

Proof. Form the theory $T_{A}$ by expanding $T$ with countably many constants for the elements of $A$. The assumptions of the previous fact are satisfied for $T_{A}$ since $A$ is good, so there exists a countable atomic model $M(A)$ for $T_{A}$. It is easy to see that the reduct of $M(A)$ to the original language is a primary model over $A$.

We will find several equivalent properties for good sets in a few more lemmas culminating in Corollary 2.8 .

Lemma 2.5. Let $A$ be a countable atomic set. If $\mathrm{S}_{\text {at }}(A)$ is countable, then $A$ is good.

Proof. Suppose $A$ is not good: Then there exists $\phi(x, a)$ with $a \in A$ and $\models$ $\exists x \phi(x, a)$, but no isolated extension of $\phi(x, a)$ exists in $\mathrm{S}_{\text {at }}(A)$. Thus, for each $\psi(x, b)$ with $b \in A$ with $\models \forall x(\psi(x, b) \rightarrow \phi(x, a))$, there is $b^{\prime} \in A$ such that $\psi(x, b)$ has at least two extensions in $\mathrm{S}_{\text {at }}\left(a b b^{\prime}\right)$. We will use this to contradict the countability of $\mathrm{S}_{\mathrm{at}}(A)$, in a similar way to the proof of boundedness of the rank.

Let $A=\left\{a_{i}: i<\omega\right\}$. We construct $\psi_{\eta}\left(x, b_{\eta}\right)$ for $\eta \in<\omega^{<}$such that $\psi_{\langle\rangle}\left(x, b_{\langle\rangle}\right)=\phi(x, a)$, if $\eta<\nu$ then $=\forall x\left(\psi_{\eta}\left(x, b_{\eta}\right) \rightarrow \psi_{\nu}\left(x, b_{\nu}\right)\right)$, each $\psi_{\eta}\left(x, b_{\eta}\right)$ isolates a complete type over $b_{\eta}, b_{\eta}$ contains $a_{i}$ if $\ell(\eta)>i$, and $\psi_{\eta^{\wedge} 0}\left(x, b_{\eta \wedge 0}\right)$ and $\psi_{\eta^{\wedge} 1}\left(x, b_{\eta^{\wedge} 1}\right)$ are contradictory. This is possible and implies that $\mathrm{S}_{\mathrm{at}}(A)$ has size continuum.

Lemma 2.6. If $M \in \mathcal{K}$ is countable and $M c$ is atomic, then $M c$ is good.

Proof. For each $\operatorname{tp}(d / M c) \in \mathrm{S}_{\text {at }}(M c)$, consider $\operatorname{tp}(d c / M) \in \mathrm{S}_{\text {at }}(M)$. It is easy to see that this induces an injection from $\mathrm{S}_{\mathrm{at}}(M c)$ into $\mathrm{S}_{\mathrm{at}}(M)$. Hence $\mathrm{S}_{\mathrm{at}}(M c)$ is countable, since $\mathrm{S}_{\text {at }}(M)$ is countable by $\omega$-stability of $\mathcal{K}$. Hence, $M c$ is good.

We now consider the dual notion to prime models. We say that $N$ is universal over $A$, if $A \subseteq N$, and for $M \in \mathcal{K}$ with $A \subseteq M$ and $\|M\|=\|N\|$, there exists an elementary map $f: M \rightarrow N$ which is the identity on $A$.

Lemma 2.7. If $M \in \mathcal{K}$ is countable, then there exists a countable universal model $N \in \mathcal{K}$ over $M$.

Proof. Let $\left(M_{n}: n<\omega\right)$ be an increasing sequence of countable models such that $M_{0}=M$ and $M_{n+1}$ realises all types in $\mathrm{S}_{\text {at }}\left(M_{n}\right)$. We could do this at once using $\mathfrak{C}$, but $\mathfrak{C}$ is not necessary here: Let $\left\{p_{i}: i<\omega\right\}$ be an enumeration of $\mathrm{S}_{\text {at }}\left(M_{n}\right)$. Let $a_{0}$ realise $p_{0}$ (which exists since $M_{i}$ is countable) and let $M_{0}^{\prime}$ be primary over $M_{n} a_{0}$, which exists by the three previous lemmas. Since $p_{1}$ is stationary, there 
exists a unique free extension $q_{1}$ over $M_{0}^{\prime}$. Let $a_{1}$ realise $q_{1}$. Let $M_{1}^{\prime}$ be primary over $M_{0}^{\prime} a_{1}$. Continue like this inductively. Let $M_{n+1}=\bigcup_{i<\omega} M_{i}^{\prime}$.

Let $N=\bigcup_{n<\omega} M_{n}$. We claim that $N$ is universal over $M$. Let $M^{\prime} \in \mathcal{K}$ be countable such that $M \prec M^{\prime}$. Write $M^{\prime}=\left\{a_{i}: i<\omega\right\}$. We construct an increasing sequence of elementary maps

$$
f_{i}: M \cup\left\{a_{0}, \ldots, a_{i}\right\} \rightarrow N,
$$

which is the identity on $M$ for $i<\omega$. This is enough as $\bigcup_{i<\omega} f_{i}$ is an elementary map sending $M^{\prime}$ into $N$, which is the identity on $M$.

Let us now construct the $f_{i}$ s. For $i=0$, let $b_{0}$ realise $\operatorname{tp}\left(a_{0} / M\right) \in \mathrm{S}_{\text {at }}(M)$ which exists in $N$ by construction, and let $f_{0}$ be the partial elementary map from $M a_{0}$ which is the identity on $M$ and sends $a_{0}$ to $b_{0}$. Having constructed $f_{i}$, let $M^{*}$ be a primary model over $M \cup\left\{a_{0}, \ldots, a_{i}\right\}$ which exists, since $M \cup\left\{a_{0}, \ldots, a_{i}\right\}$ is good. There exists $k<\omega$ such that $a_{0}, \ldots, a_{i} \in M_{k}$. By definition, we can extend $f_{i}$ to $f_{i}^{*}: M^{*} \rightarrow M_{k}$, which is the identity on $M$. Then the image of $\operatorname{tp}\left(a_{i+1} / M^{*}\right)$ under $f_{i}^{*}$ can be extended to a type in $\mathrm{S}_{\mathrm{at}}\left(M_{k}\right)$ (by stationarity), which is then realised by some element $b_{i+1}$ of $M_{k+1}$. Let $f_{i+1}$ be the partial elementary map extending $f_{i}$ and sending $a_{i+1}$ to $b_{i+1}$. This finishes the construction.

So, we finally have:

Corollary 2.8. Let $A$ be a countable atomic set. The following conditions are equivalent:

(1) A is good.

(2) There is a primary model over A.

(3) There is a countable universal model over $A$.

(4) $\mathrm{S}_{\mathrm{at}}(A)$ is countable.

Proof. We showed (1) implies (2) and (4) implies (1). For (2) implies (3): Let $M$ be primary over $A$. By the previous lemma there exists $N$ universal over $M$. This implies immediately that $N$ is universal over $A$. (3) implies (4) is clear: Let $N$ be universal over $A$. Each $p \in \mathrm{S}_{\text {at }}(A)$ is realised in some countable model $M_{p}$, which embeds in $N$ over $A$ by universality of $N$. Hence, each $p \in \mathrm{S}_{\text {at }}(A)$ is realised in $N$ so $\left|\mathrm{S}_{\text {at }}(A)\right| \leq\|N\|$.

Unless we are in the homogeneous case, there are countable atomic sets $A$ such that $\mathrm{S}_{\text {at }}(A)$ is uncountable, so some sets are good and others are not. The next remark follows by counting types.

Remark 2.9. Let $A$ be countable and good. Let $c$ realise $p \in \mathrm{S}_{\text {at }}(A)$. Then $A c$ is good. 
We pointed out in a previous section that a type $p \in \mathrm{S}_{\text {at }}(A)$ may fail to have an extension in $\mathrm{S}_{\mathrm{at}}(M)$ where $M$ contains $A$; this only happens when $A$ is not good. In fact, it follows easily from the next lemma and $\omega$-stability of $\mathcal{K}$ that a countable set $A$ is good if and only if each type in $\mathrm{S}_{\text {at }}(A)$ extends to a type over $M$ for any $M$ containing $A$.

Lemma 2.10. Let $A$ be atomic. Let $B \subseteq A$ be a countable good set. Let $p \in$ $\mathrm{S}_{\mathrm{at}}(B)$. Then there is $q \in \mathrm{S}_{\mathrm{at}}(A)$ extending $p$.

Proof. Let $M$ be a primary model over $B$. Let $c \models p$ and let $M^{\prime}$ be a primary model over $B c$ (which exists by the previous remark). Without loss of generality $M \prec M^{\prime}$. Hence $\operatorname{tp}(c / M) \in \mathrm{S}_{\text {at }}(M)$ is an extension of $p$. Define $q \in \mathrm{S}_{\text {at }}(A)$ extending $p$, using $\operatorname{tp}(c / M)$, just as in the proof of Proposition 1.5.

We finish this section with the problem of existence and uniqueness of countable full models over countable sets.

Proposition 2.11. Let $A$ be countable and atomic. Then there exists a countable $M \in \mathcal{K}$ which is full over $A$.

Proof. Let $M_{0}$ be any countable model containing $A$. Let $M_{n+1}$ be countable realising each type in $\mathrm{S}_{\text {at }}\left(M_{n}\right)$. Let $N=\bigcup_{n<\omega} M_{n}$. Then $N \in \mathcal{K}$ is countable and contains $A$. We claim that $N$ is full over $A$. Let $p \in \mathrm{S}_{\text {at }}(A c)$ be the unique free extension of a stationary type in $\mathrm{S}_{\text {at }}(c)$. There is $n<\omega$ such that $c \in M_{n}$, so $A c \subseteq M_{n}$. There is a unique free extension of $p$ in $\mathrm{S}_{\text {at }}\left(M_{n}\right)$, and this extension is realised in $M_{n+1}$, hence in $N$.

For uniqueness, $A$ needs to be good.

Proposition 2.12. Suppose that $A$ is good and countable. Suppose that $M$ and $N \in \mathcal{K}$ are countable and full over $A$. Then $M$ is isomorphic to $N$ over $A$.

Proof. Let $M=A \cup\left\{a_{i}: i<\omega\right\}$ and $N=A \cup\left\{b_{i}: i<\omega\right\}$. We construct an increasing and continuous sequence of partial elementary maps $f_{i}: M \rightarrow N$ such that $\operatorname{dom}\left(f_{i}\right)=A_{i}, \operatorname{ran}\left(f_{i}\right)=B_{i}, f_{i}\left\lceil A=i d_{A}\right.$ and $a_{i} \in A_{2 i}$ and $b_{i} \in B_{2 i+1}$, $A_{i} B_{i} \backslash A$ is finite.

This is clearly enough. Let us see that this is possible. We first construct $f_{0}$. Since $A$ is good, there is a primary model $M^{\prime}$ over $A$. Without loss of generality $M^{\prime} \prec M$. There is an elementary map $f: M^{\prime} \rightarrow N$, which is the identity on $A$. Consider the stationary type $\operatorname{tp}\left(a_{0} / M^{\prime}\right)$. It is the unique free extension of the stationary type $p=\operatorname{tp}\left(a_{0} / A c\right)$ for some $c \in M^{\prime}$. The image of $p$ under $f$ is realised in $N$ by some $b$, by fullness of $N$, and $\operatorname{tp}\left(a_{0} / A\right)=\operatorname{tp}(b / A)$. Let $f_{0}$ be the map extending $i d_{A}$ sending $a_{0}$ to $b$. 
Let $M^{\prime \prime}$ be primary over $A b$ ( $M^{\prime \prime}$ exists since $A$ is good and $A b$ is atomic). There exists $g: M^{\prime \prime} \rightarrow M$ extending the inverse of $f_{0}$. The stationary type $\operatorname{tp}\left(b_{0} / M^{\prime \prime}\right)$ is the unique extension of some $q \in \mathrm{S}_{\text {at }}(A b d)$, with $d \in M^{\prime \prime}$. The image of $q$ under $g$ is realised in $M$ by fullness of $M$ over $A$, so the map $f_{1}$ extending $f_{0}$ sending a realisation of $g(q)$ to $b_{0}$ is elementary.

Now assume that $f_{2 i+1}$ has been constructed. Then $A_{2 i+1} \backslash A$ is finite, so $A_{2 i+1}$ is a good set containing $A$. Hence, there is a primary model $M^{\prime}$ containing $A_{2 i+1}$. The type $\operatorname{tp}\left(a_{i+1} / M^{\prime}\right)$ is stationary and is the unique free extension of a type $p=\operatorname{tp}\left(a_{i+1} / A_{2 i+1} c\right)$ for some $c \in M^{\prime}$. There is $f: M^{\prime} \rightarrow N$ extending $f_{2 i+1}$. The image of $p$ under $f$ is realised in $N$, which allows us to find $f_{2 i+2}$ with domain $A_{2 i+1} \cup a_{i+1}$, extending $f_{2 i+1}$. The construction of $f_{2 i+3}$ is similar.

\section{INDEPENDENT SYSTEMS, EXCELLENCE, AND CATEGORICITY THEOREM}

To motivate the definition of excellence, let us consider the problem of existence and uniqueness of uncountable full models. For full models of size $\aleph_{1}$ over a countable (good) set, it is still manageable. For existence: simply iterate $\omega_{1}$ times the construction of Proposition 2.11 (or, under our assumption, to consider an appropriate submodel of $\mathfrak{C})$. For uniqueness: To show that $M, N \in \mathcal{K}$ of size $\aleph_{1}$, full over the good countable set $A$, are isomorphic, choose $\left(M_{i}: i<\omega_{1}\right)$, and $\left(N_{i}: i<\omega_{1}\right)$ increasing and continuous chain of countable models such that $\bigcup_{i<\omega_{1}} M_{i}=M$ and $\bigcup_{i<\omega_{1}} N_{i}=N$, with $M_{0}$ and $N_{0}$ primary over $A, M_{i+1}$ full over $M_{i}$ and $N_{i+1}$ full over $N_{i}$. The isomorphism between $M$ and $N$ is then defined inductively by using Proposition 2.12.

However, to prove the existence or uniqueness of uncountable full models over larger sets (for example over a model of size $\aleph_{1}$ ), or to prove the existence and uniqueness of full models of size at least $\aleph_{2}$ is more problematic. The key ingredient in both proofs is the existence of a primary model over $M a$, where $M \in$ $\mathcal{K}$ countable and $M a$ is atomic. We have not proved this for $M \in \mathcal{K}$ uncountable. Here is a possible strategy to prove this for $M$ of size, say, $\aleph_{1}$ :

Choose $\left(M_{i}: i<\omega_{1}\right)$ a resolution of $M$. There exists a primary model $N_{0}$ over $M_{0} a$, since $M_{0} a$ is good. Suppose $N_{0} \cup M_{1}$ is atomic and good. Then we could find $N_{1}$ primary over $N_{0} \cup M_{1}$. Inductively, if $N_{i} \cup M_{i+1}$ is atomic and good for each $i<\omega_{1}$, then we could continue this process, and by taking unions at limit, obtain an increasing and continuous chain of models $\left(N_{i}: i<\omega_{1}\right)$ such that $N_{i+1}$ is primary over $N_{i} \cup M_{i+1}$. The hope is then that, by pasting together the constructions, one could show that $\bigcup_{i<\omega_{1}} N_{i}$ is primary over $M a$. To help us carry out this construction, we will use independence. First choose $M_{0}$ countable such that, in addition, $a$ is free from $M$ over $M_{0}$; this, we will see, ensures that the primary model $N_{0}$ is free from $M$ over $M_{0}$ and $N_{0} \cup M$ is atomic. Inductively, assume that $N_{i}$ is free from $M$ over $M_{i}$ and $N_{i} \cup M$ is atomic. Then, if $N_{i} \cup M_{i+1}$ is good, we can find $N_{i+1}$ primary over $N_{i} \cup M_{i+1}$ such that, in addition, $N_{i+1}$ is 
free from $M$ over $N_{i+1}$ and $N_{i+1} \cup M$ is atomic. Taking unions at limit allows us to construct $N=\bigcup_{i<\omega_{1}} N_{i}$ (provided $N_{i} \cup M_{i+1}$ is good at each stage), and now, it is easy to see that $N$ is primary over $M a$. Thus, the problem of finding a primary model over $M a$ is reduced to finding primary models over countable sets of the form $M_{1} \cup M_{2}$, where $M_{1}$ is free from $M_{2}$ over $M_{0}$, and $M_{1} \cup M_{2}$ is atomic. The gain is that the models involved are countable; the cost is that we have to consider 2-dimensional (independent) systems: $N_{i+1}$ completes a square whose vertices are $M_{i}, M_{i+1}$, and $N_{i}$ and edges given by the relation $\prec$.

Now consider $M a$, with $M \in \mathcal{K}$ with $M a$ atomic, but this time with $M$ of size $\aleph_{2}$. Using the same idea leads us to ask about the existence of primary models over the atomic set $M_{1} \cup M_{2}$, where $M_{0}, M_{1}, M_{2} \in \mathcal{K}, M_{0} \prec M_{1}, M_{2}, M_{1}$ is free from $M_{2}$ over $M_{0}$, and $M_{\ell}$ has size $\aleph_{1}$ for $\ell=0,1,2$, i.e. an independent 2 -dimensional system of models of size $\aleph_{1}$. We can repeat the same procedure to analyse this 2 -dimensional system. Write a resolution $\left(M_{\ell}^{i}: i<\omega_{1}\right)$ of countable models for each $M_{\ell}, \ell=0,1,2$. We can try to construct the primary model over $M_{1} \cup M_{2}$ as the union of an increasing and continuous chain of models $\left(N_{i}: i<\right.$ $\left.\omega_{1}\right)$ such that $N_{0}$ is primary over $M_{1}^{0} \cup M_{2}^{0}$, and $N_{i+1}$ primary over $M_{1}^{i+1} \cup M_{2}^{i+1} \cup$ $N_{i}$. To carry this out, we need to ensure that the countable sets $M_{1}^{i+1} \cup M_{2}^{i+1} \cup N_{i}$ are atomic and good at each stage $i<\omega_{1}$; here again independence will play an important part. In all, the gain is that the models are now countable, but the cost is that we have to consider 3-dimensional systems: $N_{i+1}$ completes the cube whose 7 other vertices are $N_{i}, M_{1}^{i}, M_{2}^{i}, M_{0}^{i}$, and $M_{1}^{i+1}, M_{2}^{i+1}$, and $M_{0}^{i+1}$, again edges are given by $\prec$. We formalise these ideas next.

We consider the following partial order $\mathcal{P}^{-}(n):=\mathcal{P}(n) \backslash n$ with respect to inclusion. We write $s \subset t$, if $s$ is a strict subset of $t$, so $\mathcal{P}^{-}(n)=\{s: s \subset n\}$, where $n=\{0,1, \ldots, n-1\}$. Then, $\mathcal{P}^{-}(0)$ is empty, $\mathcal{P}^{-}(1)$ is a point, $\mathcal{P}^{-}(2)$ is a square without one of its vertices, $\mathcal{P}^{-}(3)$ is a cube without one its vertices, and so forth.

Definition 3.1. An independent $(\lambda, n)$-system is a collection of models

$$
\left(M_{s}: s \subset n\right)
$$

such that

(1) Each $M_{s} \in \mathcal{K}$ has size $\lambda$.

(2) $M_{s} \prec M_{t}$ if $s \subset t$.

(3) The set $A_{s}=\bigcup_{t \subset n} M_{t}$ is atomic.

(4) For each $s, M_{s} \underset{A_{s}}{\downarrow} B_{s}$, where $B_{s}=\bigcup_{t \not \subset s} M_{t}$.

We will omit the parameters when they are either obvious or not important.

The next definition is not formally made in [Sh 87b]. Also, in [Sh 87b], $(\lambda, n)$-existence refers to a different property. 
Definition 3.2. We say that $\mathcal{K}$ has $(\lambda, n)$-existence if there exists a primary model over $\bigcup_{s \subset n} M_{s}$, for each $(\lambda, n)$-independent system $\left(M_{s}: s \subset n\right)$.

Thus, in this paper, $\left(\aleph_{0}, n\right)$-existence is equivalent to the requirement that $\bigcup_{s \subset n} M_{s}$ is good for any independent $\left(\aleph_{0}, n\right)$-system. The next definition is the main definition of this paper.

Definition 3.3. $\mathcal{K}$ is excellent if $\mathcal{K}$ has $\left(\aleph_{0}, n\right)$-existence, for each $n<\omega$.

We will now show how the existence of primary models over some (countable) sets implies the existence of primary models over other sets. If we had primary models over all countable atomic sets, then we would have them over all atomic sets [Le2].

Fact 3.4 (Lessmann). Assume that $\mathcal{K}$ is $\omega$-stable and has an uncountable model. The following are equivalent:

(1) There is a primary model over each countable atomic set.

(2) There is a primary model over each atomic set.

(3) There are arbitrarily large homogeneous models.

In our case, the situation is a bit more delicate. We now prove a lemma, which we refer to as dominance.

Lemma 3.5. Suppose that $A \downarrow B$, where $M \in \mathcal{K}$ and $A B M$ is an atomic set. Suppose that $M(A)$ is primary over $M A$. Then $M(A) \downarrow B$. $M$

Proof. By finite character of independence, it is enough to show this for $A$ and $B$ finite. Write $A=a$ and $B=b$. It is also enough to show that if $\operatorname{tp}(c / M a)$ is isolated and $a \underset{M}{\downarrow} b$, then $c \underset{M}{\downarrow} a b$. But this is clear since $\operatorname{tp}(c / M a)$ isolates $\operatorname{tp}(c / M a b)$ (as $M$ is a model).

We now formalise the proof discussed at the beginning of this section with two theorems. The first shows that the existence of primary models over sets of the form $M a$ does indeed follow from the existence of primary models over independent 2-dimensional systems of models of smaller size.

Theorem 3.6. Suppose that $\mathcal{K}$ has $(\mu, 2)$-existence, for each $\mu<\lambda$. Then, if $M \in \mathcal{K}$ has size $\lambda$ and $M a$ is atomic, there exists a primary model over $M a$.

Proof. Let $B$ be a finite subset of $M$ such that $c \underset{B}{\downarrow} M$. Choose an increasing and $B$

continuous sequence of models $\left(M_{i}: i<\lambda\right)$, such that $M_{i} \in \mathcal{K}, B \subseteq M_{i}$, $\left\|M_{i}\right\| \leq|i|+\aleph_{0}$, and $\bigcup_{i<\lambda} M_{i}=M$. 
Now construct an increasing and continuous sequence of models

$$
\left(N_{i}: i<\lambda\right)
$$

such that $N_{i} \downarrow M, N_{i} \cup M$ is atomic, $N_{0}$ is primary over $M_{0} a$, and $N_{i+1}$ is $M_{i}$ primary over $N_{i} \cup M_{i+1}$.

For $i=0$, a primary model $N_{0}$ over $M_{0} a$ exists, since $M_{0}$ is countable (and so $M_{0} a$ is good). By independence calculus, we may assume that $M \cup N_{0}$ is atomic and $N_{0} \downarrow M$. Hence, $\left(M_{0}, N_{0}, M_{1}\right)$ form an independent $\left(\aleph_{0}, 2\right)$-system. $M_{0}$

Inductively, notice that $\left(M_{i}, N_{i}, M_{i+1}\right)$ forms an independent $\left(|i|+\aleph_{0}, 2\right)$-system. By $\left(|i|+\aleph_{0}, 2\right)$-existence, there exists $N_{i+1}$ primary over $M_{i+1} \cup N_{i}$. By induction hypothesis and monotonicity, $N_{i} \underset{M_{i+1}}{\downarrow} M$, so $N_{i} \cup M_{i+1} \underset{M_{i+1}}{\downarrow} M$. Thus $N_{i+1} \underset{M_{i+1}}{\downarrow} M$ by dominance. At limits, take the union and notice that the independence and atomicity follows by finite character of these notions.

Let $N=\bigcup_{i<\lambda} N_{i}$. Then, by pasting the constructions together and using independence, it is not difficult to see that $N$ is primary over $M a$.

The next theorem states that the same principle extends to larger dimensions.

Theorem 3.7. Let $\lambda$ be an infinite cardinal and $n<\omega$. Suppose that $\mathcal{K}$ has $(\mu, n)$ and $(\mu, n+1)$-existence, for each $\mu<\lambda$. Then $\mathcal{K}$ has $(\lambda, n)$-existence.

Proof. We prove the particular case when $n=2$. Suppose $M_{0} \prec M_{\ell}$, for $\ell=1,2$ forms an independent $(\lambda, 2)$-system.

Choose an increasing and continuous sequences $\left(M_{\ell}^{i}: i<\lambda\right)$ of models of $\mathcal{K}$ such that $\left\|M_{\ell}^{i}\right\| \leq|i|+\aleph_{0}$, for $\ell=0,1,2$, with $\bigcup_{i<\lambda^{+}} M_{\ell}^{i}=M_{\ell}$ and

$$
M_{1}^{i} \underset{M_{0}^{i}}{\downarrow} M_{2} \quad \text { and } \quad M_{2}^{i} \underset{M_{0}^{i}}{\downarrow} M_{1} .
$$

This is done as follows: Enumerate $M_{\ell}=\left\{a_{\ell}^{i}: i<\lambda\right\}$, for $\ell=0,1,2$. For $i$ a limit, define $M_{\ell}^{i}$ by continuity. For $i=0$ or a successor, having chosen $M_{\ell}^{i}$ containing $a_{\ell}^{i}$, choose $M_{0}^{i}$ containing $a_{0}^{i}$ of size $|i|+\aleph_{0}$ such that $M_{\ell}^{0} \downarrow M_{0}$, for $\ell=1,2$. Then, we obtain (*) by transitivity, since $M_{1} \underset{M_{0}}{\downarrow} M_{2}$.

$$
M_{0}^{i}
$$

Now construct an increasing and continuous sequence of models

$$
\left(N_{i}: i<\lambda\right)
$$

such that: 
(1) $N_{0}$ is primary over $M_{1}^{0} \cup M_{2}^{0}$,

(2) $N_{i} M_{1} \cup M_{2}$ is atomic,

(3) $N_{i+1}$ is primary over $M_{1}^{i+1} \cup M_{2}^{i+1} \cup N_{i}$,

(4) $N_{i}=\bigcup_{j<i} N_{j}$, for $i$ a limit.

This is possible: For $i=0$, we use $\left(\aleph_{0}, 2\right)$-existence (atomicity is obtained by extension). At successor stage $i$, we use $\left(|i|+\aleph_{0}, 3\right)$-existence after checking that $\left(M_{\ell}^{i}, M_{\ell}^{i+1}, N_{i}: \ell=0,1,2\right)$ forms an independent $\left(|i|+\aleph_{0}, 3\right)$-system (use independence calculus and dominance, just as the previous theorem). At limit stages, we define $N_{i}$ by continuity (again, atomicity is preserved and so is independence).

This is enough, as $\bigcup_{i<\lambda} N_{i}$ is primary over $M_{1} \cup M_{2}$ (pasting the constructions and using independence).

We now show two theorems showing that excellence implies the existence of primary models also over uncountable sets (this can be further extended to other systems of models [GrHa]).

Theorem 3.8. Suppose that $\mathcal{K}$ is excellent. Then, $\mathcal{K}$ has $(\lambda, n)$-existence for all $n<\omega$ and for all cardinals $\lambda$.

Proof. We prove this by induction on $\lambda$ for all $n<\omega$. For $\lambda=\aleph_{0}$, this is the definition of excellence. Assume now that $\lambda>\aleph_{0}$, and that $(\mu, n)$-existence holds for each $\mu<\lambda$ and for all $n<\omega$. Then $(\lambda, n)$-existence follows from Theorem 3.7.

We can finally prove:

Theorem 3.9. Suppose that $\mathcal{K}$ is excellent. Then for any $M \in \mathcal{K}$ and a such that $M a$ is atomic, there exists a primary model over $M a$.

Proof. By Theorems 3.6 and 3.8.

The previous theorem was the key idea behind extending the proof of the existence of full models to higher cardinalities. Before doing this, we prove a lemma.

Lemma 3.10. Assume that $\mathcal{K}$ is excellent. Suppose that $\left(M_{i}: i<\lambda\right)$ is an increasing and continuous chain of models in $\mathcal{K}, p \in S(C)$ is stationary with $C \subseteq M_{0}$, and for each $i<\lambda, a_{i} \in M_{i+1} \backslash M_{i}$ and the type $\operatorname{tp}\left(a_{i} / M_{i}\right)$ is the unique free extension of $p$. Let $I=\left(a_{i}: i<\lambda\right)$. Then

(1) I is indiscernible over $M_{0}$.

(2) For each $b \in M_{\lambda}$, there is a finite set $J \subseteq I$ such that $I \backslash J$ is indiscernible over $b$. 
Proof. Indiscernibility over $M_{0}$ is clear. Let us prove (2). Construct $\left(N_{i}: i<\lambda\right)$ increasing and continuous such that $N_{i} \prec M_{i}$ and $N_{0}$ is countable and atomic, and $N_{i+1}$ is primary over $N_{i} \cup a_{i}$. This is possible by excellence. Let $N=\bigcup_{i<\lambda} N_{i} \prec$ $M$. Then $N$ is primary over $N_{0} \cup\left\{a_{i}: i<\lambda\right\}$.

Let $b \in M_{\lambda}$. Then there is $c \in N$ and $i_{1}<\ldots i_{n}<\lambda$ such that $\operatorname{tp}(b / N)$ does not split over $c$. Now $\operatorname{tp}\left(c / N_{0}\left\{a_{i}: i<\lambda\right\}\right)$ is isolated by a formula $\phi\left(x, d, a_{i_{1}}, \ldots, a_{i_{n}}\right)$, where $d \in N_{0}$ and $i_{1}<\cdots<i_{n}<\lambda$.

We claim that $I \backslash\left\{a_{i_{1}}, \ldots, a_{i_{n}}\right\}$ is indiscernible over $b$. Let $\bar{a}, \bar{a}^{\prime}$ two finite subsequences of $I \backslash\left\{a_{i_{1}}, \ldots, a_{i_{n}}\right\}$ of the same length. Notice that

$$
\operatorname{tp}\left(\bar{a} / d a_{i_{1}}, \ldots, a_{i_{n}}\right)=\operatorname{tp}\left(\bar{a}^{\prime} / d a_{i_{1}}, \ldots, a_{i_{n}}\right),
$$

since $d \in N_{0}$ and $I$ is indiscernible over $N_{0}$. Since $\operatorname{tp}(c / N)$ is isolated over $d a_{i_{1}}, \ldots, a_{i_{n}}$, then necessarily $\operatorname{tp}(\bar{a} / c)=\operatorname{tp}\left(\bar{a}^{\prime} / c\right)$. By nonsplitting, it follows that $\operatorname{tp}(\bar{a} b / \emptyset)=\operatorname{tp}\left(\bar{a}^{\prime} b / \emptyset\right)$. This shows that $I \backslash\left\{a_{i_{1}}, \ldots, a_{i_{n}}\right\}$ is indiscernible over $b$.

In general, indiscernible sequences in an excellent class cannot be extended; however those obtained as above can. This gives us a way of extracting an extensible indiscernible sequence from any uncountable set (see the proof of categoricity for more details). We can now construct full models directly. The next theorem is much stronger than our Hypothesis 0.4. There we assumed the existence of some suitably large full model; here we show that every model extends to a full model.

Theorem 3.11. Suppose that $\mathcal{K}$ is excellent. Let $M \in \mathcal{K}$. Then there is a full model $N$ over $M$ of size $\lambda$ for any $\lambda \geq\|M\|$.

Proof. Let $M \in \mathcal{K}$ be given and let $\lambda \geq\|M\|$. We construct an increasing and continuous sequence of models $\left(M_{i}: i<\lambda\right)$ such that $M_{0}=M,\left\|M_{i}\right\|=|i|+\aleph_{0}$, and $M_{i+1}$ realises all types in $\mathrm{S}_{\text {at }}\left(M_{i}\right)$. This is done as in the countable case using excellence: Having constructed $M_{i}$ of size at most $\lambda$, by $\omega$-stability, $\mathrm{S}_{\text {at }}\left(M_{i}\right)=$ $\left\{p_{j}: j<\lambda\right\}$ (since $\mathcal{K}$ is $\lambda$-stable by Proposition 1.6). Construct an increasing and continuous sequence of models $M_{j}^{\prime}$, such that $M_{j+i}^{\prime}$ is primary over $M_{j}^{\prime} a_{j}$, and $a_{j}$ realises the unique free extension of $p_{j}$ over $M_{j}^{\prime}$. This is possible by stationarity of each $p_{j}$ and the fact that a primary model exists over each set of the form $M a$, where $a$ realises a type in $\mathrm{S}_{\mathrm{at}}(M)$.

Let $M=\bigcup_{i<\lambda} M_{i}$. We claim that $M$ is full. Let $p \in S(C)$ with $C \subseteq M$ of size less than $\lambda$, be stationary. Let $c \in C$, such that $p$ does not split over $c$. Without loss of generality, we may assume that $c \in M_{0}$. Let $I=\left\{a_{i}: i<\lambda\right\}$ be such that $a_{i} \in M_{i+1}$ realises $p\left\lceil M_{i}\right.$. The $a_{i}$ s exist by construction. Now, if $a_{i}=a_{j}$ for $i<j$, then $p$ is realised by $a_{i}$. Otherwise, $I$ has size $\lambda$. By the previous fact, $I$ is an indiscernible sequence and there exists $J \subseteq I$ of size less than $\lambda$, such 
that $I \backslash J$ is indiscernible over $C$. Thus, each element of $I \backslash J$ realises $p$, so $p$ is realised in $M$.

We now consider the problem of uniqueness of full models. Similarly to the previous theorem, we could prove uniqueness of full models like Proposition 2.12 by using Theorem 3.9. Instead, we illustrate again this idea of decomposing a certain problem into a larger dimensional problem involving smaller models. For this, we define full independent systems. We could have used this idea to construct full models also.

Definition 3.12. A $(\lambda, n)$-independent system is called a full $(\lambda, n)$-independent system if $M_{s}$ is full over $A_{s}$ for each $s \subset n$.

Definition 3.13. We say that $\mathcal{K}$ satisfies $(\lambda, n)$-uniqueness, if there is a unique full model over $A_{n}$, for any full $(\lambda, n)$-independent system $\left(M_{s}: s \subset n\right)$.

Lemma 3.14. If $\mathcal{K}$ is excellent, then $\mathcal{K}$ satisfies $\left(\aleph_{0}, n\right)$-uniqueness, for all $n<\omega$.

Proof. By excellence, the set $\bigcup_{s \subset n} M_{s}$ is good, for any $\left(\aleph_{0}, n\right)$-independent system $\left(M_{s}: s \subset n\right)$. There is a full model over it, and by Proposition 2.12, the full model over it is unique.

Proposition 3.15. Assume that $\mathcal{K}$ is excellent. If $\mathcal{K}$ has $(\mu, n)$ and $(\mu, n+1)$ uniqueness, for each $\mu<\lambda$, then $\mathcal{K}$ has $(\lambda, n)$-uniqueness.

Proof. We prove this for $n=1$. Suppose that $M_{\ell}$ is full over $M_{0}$ for $\ell=1,2$ and $M_{\ell} \in \mathcal{K}$ have size $\lambda$, and $M_{0}$ is full. We must show that $M_{1}$ is isomorphic to $M_{2}$ over $M_{0}$.

We construct three increasing and continuous chains of models

$$
\left(M_{\ell}^{i}: i<\lambda\right) \text {, }
$$

with $\left\|M_{\ell}^{i}\right\| \leq|i|+\aleph_{0}$, such that $M_{\ell}=\bigcup_{i<\lambda} M_{\ell}^{i}$, for $\ell=0,1,2$ and the following conditions hold:

(1) $M_{0}^{i+1}$ is full over $M_{0}^{i}$;

(2) $M_{\ell}^{i}$ is full over $M_{0}^{i}$ for $\ell=1,2$;

(3) $M_{\ell}^{i+1}$ is full over $M_{\ell}^{i} \cup M_{0}^{i+1}$;

(4) $M_{\ell}^{i+1} \cup M_{0}^{i+1}$ is atomic, for $\ell=1,2$;

(5) $M_{\ell}^{i} \downarrow M_{0}^{i+1}$, for $\ell=1,2$.

$$
M_{0}^{i}
$$

This is possible: For $i=0$, to reconcile (1) and (2) with (5), we construct $M_{\ell}^{0}$ for $\ell=0,1,2$ by taking $\omega$-chains of models. Choosing first the approximation to $M_{\ell}^{0, n} \prec M_{\ell}$ which are full over $M_{0}^{0, n-1}$ (for $\ell=1,2$, and then $M_{0}^{0, n+1} \prec M_{0}$ so $M_{\ell}^{0, n} \quad \downarrow \quad M_{0}$ and $M_{0}^{0, n+1}$ is full. The resulting union has the desired property. $M_{0}^{0, n+1}$ 
For $i$ a limit ordinal we define everything by continuity. The successor stage is similar to the base case, with chains of length $|i|+\aleph_{0}$ (see the proof of the existence of full models for details).

This is enough: Notice that by $(<\lambda, 2)$-uniqueness, the model $M_{\ell}^{i+1}$ is the unique full model over $M_{\ell}^{i} \cup M_{0}^{i+1}$ (for $\ell=1,2$ ), as the relevant systems are full. We can therefore construct an increasing and continuous sequence of isomorphisms $f_{i}: M_{1}^{i} \rightarrow M_{2}^{i}$, which are the identity on $M_{0}^{i}$, inductively. The union is the required isomorphism between $M_{1}$ and $M_{2}$ over $M_{0}$.

For $i=0$, by $\left(\aleph_{0}, 1\right)$-uniqueness, since both $M_{1}^{0}$ and $M_{2}^{0}$ are full over $M_{0}^{0}$, there is an isomorphism $f_{0}$ from $M_{1}^{0}$ to $M_{2}^{0}$, which is the identity on $M_{0}^{0}$. At limit stages, we define $f_{i}$ by continuity.

At successor stage, assume that $f_{i}$ has been constructed. Let $g$ be an elementary map extending $f_{i}$, whose domain contains $M_{1}^{i+1}$, which is the identity on $M_{0}^{i+1}$. This is possible since $M_{1}^{i} \downarrow M_{0}^{i+1}$. Now $g\left(M_{1}^{i+1}\right)$ and $M_{2}^{i+1}$ are $M_{0}^{i}$

both full over $M_{0}^{i+1} \cup M_{2}^{i}$. Hence, by $\left(|i+1|+\aleph_{0}, 2\right)$-uniqueness, $g\left(M_{1}^{i+1}\right)$ and $M_{2}^{i+1}$ are isomorphic over $M_{0}^{i+1} \cup M_{2}^{i}$. This isomorphism yields an isomorphism $f_{i+1}: M_{1}^{i+1} \rightarrow M_{2}^{i+1}$ extending $f_{i}$ which is the identity on $M_{0}^{i+1}$.

The next theorem now follows by induction on $\lambda$ for all $n<\omega$, using Lemma 3.14 and Proposition 3.15 just like in the proof of Theorem 3.8.

Theorem 3.16. Suppose that $\mathcal{K}$ is excellent. Then $\mathcal{K}$ has the $(\lambda, n)$-uniqueness property for all cardinals $\lambda$, and $n<\omega$.

The next corollary is simply $(\lambda, 0)$-uniqueness:

Corollary 3.17. For each cardinal $\lambda$, there is a unique full model $M \in \mathcal{K}$ of size $\lambda$.

At this point, it may be helpful to examine our Hypothesis again. We have now shown that $\mathfrak{C}$ functions as a monster model; by uniqueness of full models the class of (small) models of $\mathcal{K}$ corresponds exactly to the class of (small) elementary submodels of $\mathfrak{C}$. Notice, however, that, in Section 1 and 2, we have only used its $\omega$-homogeneity.

We can now present Shelah's proof of categoricity (for a Baldwin-Lachlan style proof, see [Le3]). The strategy is as follows: There exists a full model in every cardinality, so the model in the categoricity cardinal is full. Any two full models of the same size are isomorphic, so if categoricity fails in some cardinal, there exists a non full model in that cardinality, which we use to construct a non full model in the categoricity cardinal, a contradiction.

Theorem 3.18. Let $\mathcal{K}$ be excellent. Suppose that $\mathcal{K}$ is $\lambda$-categorical for some uncountable $\lambda$. Then $\mathcal{K}$ is $\mu$-categorical for all uncountable $\mu$. 
Proof. We proved that there is a unique full model up to isomorphism in each cardinal (Theorem 3.11 and Corollary 3.16).

Assume, for a contradiction, that $\mu$ is the first uncountable cardinal such that $\mathcal{K}$ is not $\mu$-categorical. Thus, there exists $M \in \mathcal{K}$ of size $\mu$ which is not full. Then, there is a stationary $p \in \mathrm{S}_{\text {at }}(c)$ for a finite $c \in M$, and $A \subseteq M$ of size $\kappa$ less than $\mu$, such that the unique free extension $q \in \mathrm{S}_{\text {at }}(A)$ extending $p$ is not realised in $M$.

Construct an increasing and continuous sequence of models

$$
\left(M_{i}: i<\kappa^{+}\right) \text {, }
$$

such that $A \subseteq M_{0}, M_{i} \prec M, M_{i+1} \neq M_{i}$. Choose $a_{i} \in M_{i+1} \backslash M_{i}$ and $b_{i} \in M_{i}$ such that $\operatorname{tp}\left(a_{i} / M_{i}\right)$ does not split over $b_{i}$, for $i<\kappa^{+}$. By Fodor's lemma, we may assume that each $b_{i} \in M_{0}$. Furthermore, by $\omega$-stability, we may assume that $\operatorname{tp}\left(a_{i} / M_{0}\right)$ is constant for all $i<\kappa^{+}$. Finally, by the pigeonhole principle, we may assume that $b_{i}=b \in M_{0}$ for all $i<\kappa^{+}$.

We have therefore a type $r \in \mathrm{S}_{\text {at }}(b)$ and $\left(a_{i}: i<\kappa^{+}\right)$such that $\operatorname{tp}\left(a_{i} / M_{i}\right)$ extends $r$ and does not split over $b$. In particular, $\left(a_{i}: i<\kappa^{+}\right)$is an indiscernible sequence.

We can now construct an increasing chain of countable models

$$
\left(N_{n}: n<\omega\right)
$$

such that $b c \in N_{0}, N_{n} \prec M_{n}, a_{n} \in N_{n+1} \backslash N_{n}$ realises the nonsplitting extension of $r$ in $\mathrm{S}_{\text {at }}\left(N_{n}\right)$. We can further choose $N_{n}$ so $N_{n}$ does not realise the type $q^{*}=$ $q \uparrow A \cap N_{0}$.

We will construct a model $N_{\lambda}$ of size $\lambda$ (the categoricity cardinal) which omits $q^{*}$. Since $q^{*}$ is the unique free extension of the stationary type $p$, and so $N_{\lambda}$ is not full, contradicting categoricity in $\lambda$.

In order to do this, we continue $\left(N_{n}: n<\omega\right)$ to obtain an increasing and continuous sequence $\left(N_{i}: i<\lambda\right)$ of models such that $a_{i} \in N_{i+1}$ realises the unique nonsplitting extension of $r$ in $\mathrm{S}_{\text {at }}\left(N_{i}\right)$ and $N_{i+1}$ is primary over $N_{i} a_{i}$, for $i \geq \omega$. This is possible by excellence, as there is a primary model over $N_{i} a_{i}$. Let $N_{\lambda}=\bigcup_{i<\lambda} N_{i}$. Then $N_{\lambda}$ has size $\lambda$ and is constructible over $N_{\omega} \cup\left\{a_{i}: i<\lambda\right\}$.

Suppose $d \in N_{\lambda}$ realises $q^{*}$. The type $\operatorname{tp}\left(d / N \cup\left\{a_{i}: i<\lambda\right\}\right) \models q^{*}$, and is isolated over $a \cup a_{i_{1}} \ldots a_{i_{m}}$, with $a \in N_{n}$ and $n+1<i_{1}<\ldots i_{m}<\lambda$. By indiscernibility of $\left(a_{i}: n \leq i<\lambda\right)$ over $N_{n}$, we may assume that $i_{1}<\cdots<$ $i_{m}<\omega$. Hence, $q^{*}$ is realised in $N_{n+1}$, a contradiction.

3.1. Shelah's original approach. In the last part of this paper, we discuss Shelah's original approach in [Sh 48], [Sh 87a], and [Sh 87b]. 
A good notion of independence, defined using $\omega$-stability, is the main prerequisite before formalising excellence. In [Sh 48], Shelah obtains $\omega$-stability from the set-theoretic assumption that $\mathbf{V}=\mathbf{L}$ and the model-theoretic assumption that $\mathcal{K}$ is categorical in $\aleph_{1}$. He does this by proving the amalgamation property over countable models. In [Sh 87a], he weakens the set-theoretic assumption to $2^{\aleph_{0}}<2^{\aleph_{1}}$, and the model-theoretic assumption to having fewer than $2^{\aleph_{1}}$ nonisomorphic models of size $\aleph_{1}$ in $\mathcal{K}$. Our hypothesis on the existence of $\mathfrak{C}$ is the substitute for amalgamation over countable models; it allows us to obtain $\omega$-stability from uncountable categoricity, within ZFC, using Ehrenfeucht-Mostowski models.

In establishing that the rank induces a good independence relation, in particular for symmetry, Shelah [Sh 48] uses a many models argument to construct $2^{\aleph_{1}}$ nonisomorphic models of size $\aleph_{1}$ from the $\omega_{1}$-order property (which follows from the failure of symmetry without any extra assumption). Here, we use $\mathfrak{C}$ to extend the construction from the $\omega_{1}$-order property to the bona fide order property, and we use it to contradict $\omega$-stability. Once these two ingredients are established, the structure part stays within ZFC and uses no further model-theoretic assumption.

For the theory of excellence per se, the reader noticed that we proved only the case $n=2$ of Theorem 3.7 (this is the only hole, as we pointed out, Proposition 3.15 is for illustrative purposes only and is not needed in proving the uniqueness of full models). To prove this for general $n$, Shelah uses the reflection principle to give a uniform method to obtain a resolution of $(<\lambda, n+1)$-independent systems whose union is any given $(\lambda, n)$-independent system. Checking that all the requirements are satisfied is then done by induction, using, among other things, the generalised symmetry lemma. This lemma states that in order to check that a system of models is independent, it is enough to check that it is independent with respect to some enumeration (preserving the order $\subset$ ). It is a generalisation of the idea that to check the independence of a sequence over a set, it is enough to check that each new element in the sequence is independent from the previous elements over the set.

In this paper, we did not address how excellence is obtained from, say, categoricity. In [Sh 87b], Shelah shows that $\left(\aleph_{0}, n+1\right)$-existence for $n \leq k$ follows from the assumptions that $\mathcal{K}$ is categorical in $\aleph_{n}$ and for each $1 \leq n \leq k$ (assuming that the $2^{\aleph_{n}}$ 's form a strictly increasing sequence). This is done by defining a strong negation of the $\left(\aleph_{0}, n\right)$-uniqueness property implying the failure of categoricity in $\aleph_{n+1}$. Hart and Shelah also showed that $\left(\aleph_{0}, n\right)$-existence does not imply $\left(\aleph_{0}, n+\right.$ 1 )-existence, and that categoricity may fail at $\aleph_{k+1}$, while holding for $\aleph_{n}, n \leq k$.

\section{REFERENCES}

[BaLa] J.T. Baldwin and A. H. Lachlan, On strongly minimal sets, Journal of Symbolic Logic, 36 (1971) 79-96.

[BuLe] Stephen Buechler and Olivier Lessmann, Journal of the AMS, 61 (2002) 91-121. 
[Gr 1] Rami Grossberg, Indiscernible sequences in a model which fails to have the order property, Journal of Symbolic Logic 56 (1991) 115-123.

[Gr 2] Rami Grossberg, On chains of relatively saturated submodels of a model without the order property, Journal of Symbolic Logic 56 (1991) 123-128.

[GrHa] Rami Grossberg and Bradd Hart, The classification theory of excellent classes, Journal of Symbolic Logic 54 (1989) 1359-1381.

[GrLe] Rami Grossberg and Olivier Lessmann, Shelah's stability spectrum and homogeneity spectrum in finite diagrams. Arch. Math. Logic 41 (2002) 1, 1-31.

[HaSh] Bradd Hart and Saharon Shelah, Categoricity over $P$ for first order $T$ or categoricity for $\phi \in L_{\omega_{1} \omega}$ can stop at $\aleph_{k}$ while holding for $\aleph_{0}, \cdots, \aleph_{k-1}$. Israel J. Math. 70 (1990), no. 2, 219-235.

[Hr] Ehud Hrushovski, Almost orthogonal regular types. Annals of Pure and Applied Logic 45 (1989) 2 139-155.

[Hy] Tapani Hyttinen, On non-structure of elementary submodels of a stable homogeneous structure Fundament Mathematicae 156 (1998) 167-182.

[HLS] Tapani Hyttinen, Olivier Lessmann, and Saharon Shelah. Interpreting groups and fields in some nonelementary classes. Preprint

[HySh] Tapani Hyttinen and Saharon Shelah, Strong splitting in stable homogeneous models, Annals of Pure and Applied Logic, 103, (2000), 201-228.

[HyLe1] Tapani Hyttinen and Olivier Lessmann, A rank for the class of elementary submodels of a superstable homogeneous model. Journal of Symbolic Logic 674 (2002) 1469-1482.

[Ke] H. Jerome Keisler, Model theory for infinitary logic , North-Holland, 1971, Amsterdam.

[KoSh] Oren Kolman and Saharon Shelah. Categoricity for $T \subseteq L_{\kappa \omega}, \kappa$ measurable. Fundamenta Mathematica

[Le1] Olivier Lessmann, Ranks and pregometries in finite diagrams, Annals of Pure and Applied Logic, 106, (2000), 49-83.

[Le2] Olivier Lessmann, Homogeneous model theory: Existence and Categoricity, in Logic and Algebra, ed. Yi Zhang, Contemporary Mathematics, Vol 302, AMS 149-164.

[Le3] Olivier Lessmann, Categoricity and U-rank in excellent classes. To appear in Journal of Symbolic Logic.

[MaSh] Michael Makkai and Saharon Shelah. Categoricity of theories in $L_{\kappa \omega}$, with $\kappa$ a compact cardinal. Annals of Pure and Applied Logic, 47:41-97, 1990.

[Ma] Leo Markus, A prime minimal model with an infinite set of indiscernibles. Israel J. of Math 11 (1972), 180-183.

[MeSh] Alan Mekler and Saharon Shelah, $L_{\infty, \omega}$-free algebras, Algebra Universalis 26 (1989), 351366.

[Mo] Michael Morley, Categoricity in power, Trans. of the A.M.S 114 (1965), pages 514-538.

[Sh 3] Saharon Shelah, Finite diagrams stable in power, Annals Math. Logic 2, (1970), pages 69118.

[Sh 12] Saharon Shelah, A combinatorial problem, stability and order in infinitary languages, Pacific Journal of Math. 41 (1972) pages 246-261.

[Sh 70] Saharon Shelah, Solution to Łoš Conjecture for uncountable languages, Notices of the A.M.S, 17 (1970), 968.

[Sh 48] Saharon Shelah, Categoricity in $\aleph_{1}$ of sentences in $L_{\omega_{1} \omega}(Q)$, Israel Journal of Math. 20 (1975), pages 127-148.

[Sh 54] Saharon Shelah, The lazy model theorist's guide to stability, Proc. of a Symp. in Louvain, March 1975, ed. P. Henrand, Logique et Analyse, 18eme annee, 71-72 (1975), pages 241-308.

[Sh 87a] Saharon Shelah. Classification theory for nonelementary classes. I. The number of uncountable models of $\psi \in L_{\omega_{1} \omega}$. Part A. Israel Journal of Mathematics, 46:212-240, 1983.

[Sh 87b] Saharon Shelah. Classification theory for nonelementary classes. I. The number of uncountable models of $\psi \in L_{\omega_{1} \omega}$. Part B. Israel Journal of Mathematics, 46:241-273, 1983. In Classification theory (Chicago, IL, 1985), volume 1292 of Lecture Notes in Mathematics, pages 
264-418. Springer, Berlin, 1987. Proceedings of the USA-Israel Conference on Classification Theory, Chicago, December 1985; ed. Baldwin, J.T.

[Sh 88] Saharon Shelah, Classification theory for nonelementary classes. II. Proceedings of the USA-Israel Conference on classification theory. Chicago (1985), J. T. Baldwin editor.

[Sh 394] Saharon Shelah, Categoricity for abstract classes with amalgamation. Annals Pure and Applied Logic 98 (1999) 261-294.

[Sh 576] Saharon Shelah. Categoricity of an abstract elementary class in two successive cardinals. Israel J. of Math., 126 (2001), 29-128.

[Sh 600] Saharon Shelah, Categoricity in abstract elementary classes. Going up an inductive step. Preprint.

[Sh 666] Saharon Shelah, On what I do not understand (and have something to say) - model theory. Fundamenta Math 166 (2000) 1-82

[Sh 705] Saharon Shelah, Toward classification theory of good $\lambda$-frames and abstract elementary classes. Preprint

[Sh a] Saharon Shelah, Classification theory and the number of nonisomorphic models, Rev. Ed., North-Holland, 1990, Amsterdam.

[ShVi] Saharon Shelah and Andres Villaveces. Toward Categoricity for Classes with no Maximal Models. Annals Pure and Applied Logic 97 (1999) 1-25.

[Zi1] Boris Zilber, Covers of the multiplicative group of an algebraically closed field of characteristic 0. Preprint.

[Zi2] Boris Zilber, Analytic and pseudo-analytic structures. Preprint.

MATHEMATICAL Institute, OXFORd UnIVERSITY, OXFORD, OX1 3LB

E-mail address: lessmann@maths.ox.ac.uk 